\title{
Pthlh, a promising cancer modifier gene in rat tongue carcinogenesis
}

\author{
HIROHIKO SUWA $^{1}$, MASATO HIRANO ${ }^{1}$, KOUJI KAWARADA ${ }^{1}$, MOTOHIKO NAGAYAMA $^{1}$, \\ MICHIKO EHARA $^{1}$, TOMONARI MURAKI ${ }^{1}$, HAYASE SHISA ${ }^{2}$, AIKO SUGIYAMA ${ }^{3}$, \\ MASAHIRO SUGIMOTO $^{3}$, HIROSHI HIAI ${ }^{3}$, MOTOO KITANO ${ }^{4}$ and JUN-ICHI TANUMA ${ }^{1}$ \\ ${ }^{1}$ Department of Oral Pathology, Division of Oral Pathogenesis and Disease Control, Asahi University School of Dentistry, \\ Mizuho, Gifu; ${ }^{2}$ Department of Pathology, Saitama Cancer Center Research Institute, Ina, Saitama; \\ ${ }^{3}$ Malignancy Control Research Laboratory, Medical Innovation Center, Graduate School of Medicine, Kyoto University, \\ Sakyo-Ku, Kyoto; ${ }^{4}$ Division of Pathology, Saitama Cooperative Hospital, Kawaguchi, Saitama, Japan
}

Received September 6, 2013; Accepted October 21, 2013

DOI: 10.3892/or.2013.2859

\begin{abstract}
Susceptibly to the induction of rat tongue cancer (TC) by oral 4-nitroquinoline 1-oxide (4NQO) exposure is a polygenic trait. Among several quantitative trait loci identified by crosses between TC-susceptible Dark Agouti (DA) rats and TC-resistant Wistar-Furth (WF) rats, we focused on tongue cancer susceptibility locus (Tcas3) of chromosome 4. We examined tongue carcinogenesis in the reciprocal congenic strains DA.WF-Tcas 3 and WF.DA-Tcas 3 and in their parental strains. The Tcas $3^{D A}$ allele, and not the Tcas $3^{W F}$ allele, significantly favored tumor latency, incidence and TC number/size. In genomic DNA of TCs induced in (DA x WF) F1 rats, the resistant Tcas $3^{W F}$ allele was frequently and selectively lost, particularly in larger tumors. Thus, we searched the possible candidate genes in the Tcas 3 region using microarray analysis of TCs in F1 rats and revealed significant upregulation of 2 cancer-related genes, parathyroid hormone-like hormone (Pthlh) and Kras2. The relevance of the WF allele of Pthlh as a cancer modifier was indicated by 3 single nucleotide polymorphisms specific to this strain. In contrast, no consistent strain-specific variations were found in Kras2. Moreover, the plasma $\mathrm{Ca}^{2+}$ level was consistently higher in DA rats when compared to the level in WF rats bearing TCs; moreover, the Pthlh-mRNA expression level was $>30$-fold higher in TCs when compared to this level in the normal tongue mucosa. Immunostaining experiments showed strong PTHrP protein expression in TCs of DA rats, and the signal was intensified in larger TCs. Kras 2 was also upregulated in TCs, but to a lesser degree than PTHrP. Thus, Pthlh is a promising candidate modifier gene in the development and progression of rat TCs.
\end{abstract}

Correspondence to: Professor Jun-Ichi Tanuma, Department of Oral Pathology, Division of Oral Pathogenesis and Disease Control, Asahi University School of Dentistry, 1851 Hozumi, Mizuho, Gifu 501-0296, Japan

E-mail: tanuma@dent.asahi-u.ac.jp

Key words: Pthlh, PTHrP, tongue cancer, speed congenic rat, microarray analysis

\section{Introduction}

In our previous research, we showed that susceptibility to 4-nitroquinoline 1-oxide (4NQO)-induced rat tongue carcinogenesis is a polygenic trait involving a number of susceptibility and resistance quantitative trait loci (QTLs) $(1,2)$. Dark Agouti (DA) rats are highly susceptible to 4NQO-induced tongue cancer (TC), whereas Wistar-Furth rats (WF) are very resistant $(3,4)$. A genome-wide association study in F2 progeny of these 2 strains revealed 5 significant QTLs, namely Tscc1-5 (Tcas1-5 in the Rat Genome Database), which account for differences in susceptibility to TC $(2,5,6)$.

In the present study, we focused on the single-locus effects of the tongue cancer susceptibility QTL 3 (Tcas3) on rat chromosome 4 (RNO4) by constructing reciprocal speed congenic strains; WF rats carrying a DA-derived Tcas3 $\left(\right.$ Tcas $\left.3^{D A}\right)$ chromosomal segment and DA rats carrying a WF-derived Tcas3 (Tcas $3^{W F}$ ) chromosomal segment. The modifier effects of Tcas 3 on $4 \mathrm{NQO}$-induced tongue carcinogenesis were confirmed by allele type-dependent incidence of TCs and frequent deletion of Tcas $3^{W F}$ in TCs in (DA x WF) F1 rats. Subsequently, we identified genes responsible for these modifying effects from the Tcas 3 region by comparing microarray analyses of TCs and normal tongue tissues, revealing a significant elevation in parathyroid hormone-like hormone (Pthlh) and Kras 2 expression. Immunohistochemistry of TCs showed that the Pthlh signal was more intense than the Kras 2 signal. Subsequent sequencing of DNA showed 3 unique single-nucleotide polymorphisms (SNP) in the WF Pthlh gene. Rats carrying the Tcas $3^{D A}$ and 4NQO-induced TCs had elevated serum Pthlh and $\mathrm{Ca}^{2+}$ levels and their WF Pthlh gene carried 3 single-nucleotide polymorphisms not found in other rat strains. These findings suggest that Pthlh is a promising candidate gene, located at Tcas3, involved in the development and progression of rat TC.

\section{Materials and methods}

Animals. The following strains of rats and their F1 (DA x WF) progeny were used in the present study. Dark Agouti/SIc rats 
were purchased from Japan SLC Inc. (Hamamatsu, Japan); Fisher 344/DuCrj (F344) rats were from Charles River Japan Inc. (Atsugi, Japan); Sprague-Dawley/JcI (SD) rats were from Japan Clea Co. (Tokyo, Japan); and DRH rats were from SEAC Yoshitomi (Fukuoka, Japan). Long Evans/Stm (LE) rats were introduced from the Saitama Cancer Center (Ina, Japan); ACI/Ms (ACI) rats were from the National Institute of Genetics (Mishima, Japan); and Donryu rats were from Osaka University (Osaka, Japan). The Wistar-Furth/Stm (WF) rats were originally from Hiroshima University (Hiroshima, Japan). The present study was carried out according to the Animal Care Guidelines of Asahi University, Gifu, Japan (10-007).

Generation of speed congenic lines. Speed congenic strains for the Tcas3 locus were developed using marker-assisted selection starting from an intercross between DA and WF rats $(7,8)$. The chromosomal segment containing Tcas3 was defined by microsatellite markers D4Rat 206 and D4Rat72. A male rat of F1 with DA-derived Tcas3 $\left(\operatorname{Tcas} 3^{D A}\right)$ from an F1 x WF cross was selected and mated with WF females $(8,9)$. After serial selective backcrossing with WF rats over 6 generations, heterozygous littermates were mated. Then a pair of littermates that were homozygous for $T c a s 3^{D A}$ were selected and subjected to successive inbreeding. The resulting strain was designated WF.DA-Tcas3. The reciprocal congenic strain DA.WF-Tcas3 was generated using the same mating protocol. All congenic rats used in the present study were of the third or subsequent generation of Tcas3 homozygotes.

Genotype analysis for speed congenic lines. All primers for PCR-based microsatellite analysis were purchased from Research Genetics, Inc. (Huntsville, AL, USA). We used 135 of the 360 polymorphic markers between DA and WF rats to characterize congenic strains. Of these, 40 were found on RNO4 and the others were distributed 10-30 cM apart on each rat chromosome $(5,6)$. Theoretically, the percentage of DA/WF segments was $<1 \%$. PCR and agarose electrophoresis of PCR products were performed as described previously $(1,2,10)$.

Tongue cancer induction. A stock solution of 4NQO (Nacalai Tesque Inc., Kyoto, Japan) at a concentration of $200 \mathrm{mg} / \mathrm{l}$ in 5\% ethanol was prepared and stored at $4^{\circ} \mathrm{C}$ until use. Starting at 6 weeks of age, all rats were given drinking water containing $0.001 \%$ 4NQO ad libitum from 5 p.m. to 9 a.m. Rats were inspected once a day for TC development and were weighed once a week. All of the rats given 4NQO (DA, WF, F1 and congenic rats) were sacrificed when they became moribund or on day 180 of the experiment. Full necropsy and histopathological examinations were performed. The diameter of the largest TC tumor (DTCmax) and the number of TCs with a diameter $\geq 5 \mathrm{~mm}$ (TC\#5) were recorded at necropsy. Paired samples of the largest TC and kidney or tail from each rat were obtained and stored at $-80^{\circ} \mathrm{C}$. High-molecular-weight DNA was extracted from the frozen tissues as previously described $(1,2)$.

Loss of heterozygosity ( $\mathrm{LOH}$ ) analysis. $\mathrm{LOH}$ in the Tcas3 region, including that of Kras2 (RGD ID: 5036392) and Pthlh (RGD ID: 11187) genes of induced TCs in F1 rats was determined using PCR-based microsatellite analysis $(1,2)$.
Fluorescently tagged primers were purchased from Applied Biosystems (Foster City, CA, USA). Positions of loci were mapped on the Rat Genome Database (6). LOH analysis was performed for tumor samples from the F1 progeny of DA and WF rats as previously described $(11,12)$. Genomic DNA from TCs of 100 reciprocal $\mathrm{F} 1$ rats (50 females and 50 males) was used to survey $\mathrm{LOH}$. Samples were scanned using a PRISM 310 Genetic Analyzer, and the data were collected automatically and analyzed using GeneScan software (both from Applied Biosystems). A Genotyper 2.0 was used to score alleles and assess LOH. A difference between the alleles was expressed as the ratio of the tumor signal to the normal signal (T1/T2 over N1/N2). Ratios $<0.67$ or $>1.35$ were considered indicative of $\mathrm{LOH}$ for that locus (12).

Microarray analysis. TCs $>5 \mathrm{~mm}$ in diameter and normal tissues from F1 rats were analyzed using microarrays. RNA was isolated from the tissues and subjected to linear amplification by RiboAmp RNA Amplification kit (Arcturus, Mountain View, CA, USA). RNA amplification efficiency was compared with that of control RNA of known quantity $(0.1 \mu \mathrm{g})$ by running in parallel with the 18 samples. Gene expression analysis was performed using the GeneChip ${ }^{\circledR}$ Rat Gene 1.0 ST Array (Affymetrix Inc., Santa Clara, CA, USA) technique according to the manufacturer's protocol. The arrays were scanned, and fluorescence intensity was measured using Microarray Suite v5.0 software (Affymetrix Inc.). The arrays were then imported into DNA-Chip analyzer software for normalization and model-based analysis. All statistical analyses were performed using S-Plus 6.0 (Insightful, Seattle, WA, USA) software. Three criteria were applied to determine differentially expressed gene transcripts as follows. First probe sets on the array that were assigned as 'absent' call in all samples. Second, a two-tailed Student's t-test was used for comparison of the average gene expression signal intensity between the F1 TCs (TC, 5-10 mm; $=15$ ) and normal samples $(n=3)$, and differences were considered significant at a critical $\alpha$ level of 0.05 . Finally, fold ratios were calculated for gene transcripts that showed a statistically significant difference $(\mathrm{P}<0.05)$, and only gene transcripts that exhibited $>2$-fold changes were included in further analyses.

Single-strand conformation polymorphism (SSCP) analysis and sequencing in F1 TC rats. To detect mutations in genes of the Tcas3 region including Pthlh and Kras2, SSCP analysis was performed in tumor samples from F1 progeny of DA and WF rats, as previously described $(12,13)$. Samples with altered electrophoretic mobility were re-amplified, and direct sequencing of both strands was performed to confirm and characterize mutations.

Quantitative real-time PCR. Total RNA was extracted from the tumors and normal tongue mucosa from 4NQO-induced TCs from $50 \mathrm{~F} 1$ rats. Tissue specimens were homogenized using an RNAqueous kit (Ambion, Grand Island, NY, USA). cDNA was generated using a High Capacity cDNA Transcription kit (Ambion) and was amplified by PCR using a TaqMan Universal PCR Master Mix and TaqMan Gene Expression assays with 18S (Rn03928990-g1), Pthlh (Rn00561818-g1) and Kras2 (Rn00580460-m1) (Applied Biosystems). PCR products 
were analyzed using Applied Biosystems StepOne ${ }^{\mathrm{TM}}$ with Gene Amp software and StepOne real-time PCR systems (13).

Direct sequence analysis of Pthlh in 8 rat strains. To detect SNPs in rat Pthlh (NC-005103.3, ID24695, M34108), genomic DNA was obtained from the rat kidney, and the entire sequence of the Pthlh locus was determined in 8 rat strains using an ABI 310 Genetic Analyzer (Applied Biosystems, Foster City, CA, USA). Allele calling was performed using the Genotyper 2.0 software (Applied Biosystems), and identified SNPs were compared with those previously reported (12-15).

To determine whether SNPs in translational regions led to structural or functional amino acid substitutions in the Pthlh protein, we conducted in silico analysis using freely available software, including MutPred (16), Panther (17), PhD-SNP (18), SNAP (19), PolyPhen2 (20) and SIFT (21). In addition, we evaluated the impact of SNPs on transcription factor-binding sites by searching for potential regulatory motifs at the 5' UTR using Matlnspector (22) and TESS (23). The software default values were used in all bioinformatic analyses.

Plasma electrolytes, serum Pthlh-related proteins and cytokines. Plasma electrolytes including $\mathrm{Ca}^{2+}, \mathrm{Na}^{+}, \mathrm{K}^{+}$, IP and $\mathrm{Cl}^{-}$were determined at Japan SLC, Inc. (24). The serum levels of Pthlh-N (normal $<3.9$ pmol/l), Pthlh-intact (normal $<1.1 \mathrm{pmol} / \mathrm{l}$ ) and Pthlh-C (normal $<55.3 \mathrm{pmol} / \mathrm{l}$ ), and interleukin (IL)-6, -8 and -11 (SRL Inc., Tokyo, Japan) were also determined (25).

Immunohistochemistry of tongue cancer. All tongue specimens from F1 rats were routinely fixed in buffered $10 \%$ formalin and were embedded in paraffin. Serial $4-\mu \mathrm{m}$ sections were dewaxed in xylene and rehydrated in graded ethanol. Sections were stained with hematoxylin and eosin (H\&E) to confirm histological diagnosis, and separate sections were used for immunohistochemical analyses. Sections were incubated with diluted rat polyclonal anti-PTHrP antibody (sc-9685; 1:100) and mouse monoclonal anti-Kras2A antibody (sc-13794; 1:50) (both from Santa Cruz Biotechnology, Inc., USA) as primary antibodies overnight at $4^{\circ} \mathrm{C}$.

Statistical analysis. Numerical data are presented as means \pm standard deviation (SD). Statistical analyses were performed using one-way ANOVA or Fisher's test with SPSS 17.0 (SPSS Inc., Chicago, IL, USA) software.

\section{Results}

Generation of speed congenic rat strains for Tcas3. To study the phenotypic effects of the single Tcas 3 locus on TC development, we established a set of speed congenic strains for Tcas 3 using marker-assisted backcrossing of DA and WF rats and generated the WF.DA-Tcas3 and DA.WF-Tcas3 strains, respectively $(8,9)$. WF.DA-Tcas 3 rats had an introgressed DA-derived RNO4 segment spanning D4Rat140 to D4Rat70, whereas DA.WF-Tcas3 rats carried a WF-derived RNO4 segment spanning D4Rat112 to D4Rat70 (Fig. 1). Both segments were $\sim 6$-cM long, were partly overlapping and contained several cancer-related genes including Pthlh and Kras2 (Fig. 1).

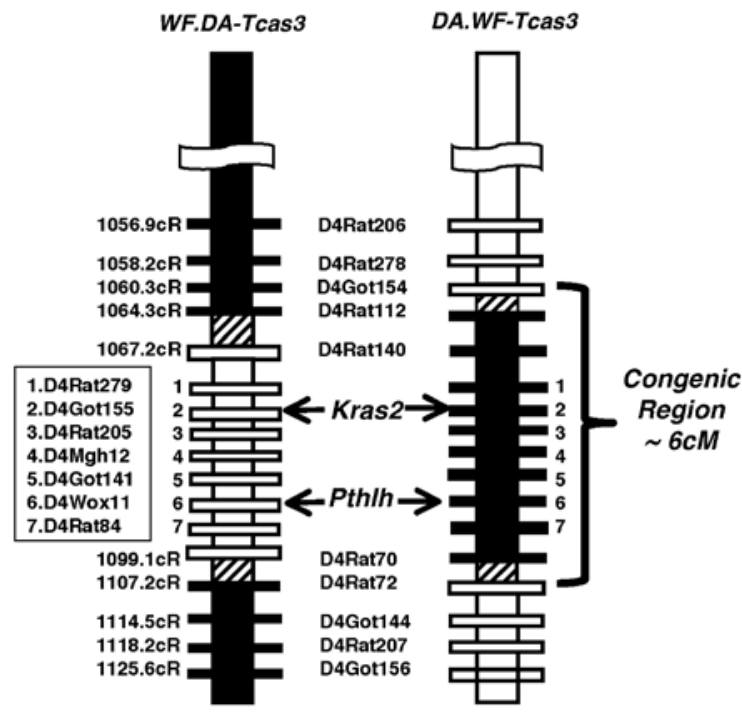

Figure 1. Tcas3 segment of RNO4 in the WF.DA-Tcas3 and DA.WF-Tcas3 rats. The closed column represents a WF-derived segment, the hatched column is undetermined and the open column represents a DA-derived segment. The closed bars represent the loci with the WF allele, and open bars represent loci with the DA allele. Numbers on the left indicate the map location of marker loci listed in the box (CR). RNO4, rat chromosome 4; WF, Wistar-Furth rats; DA, Dark Agouti rats.

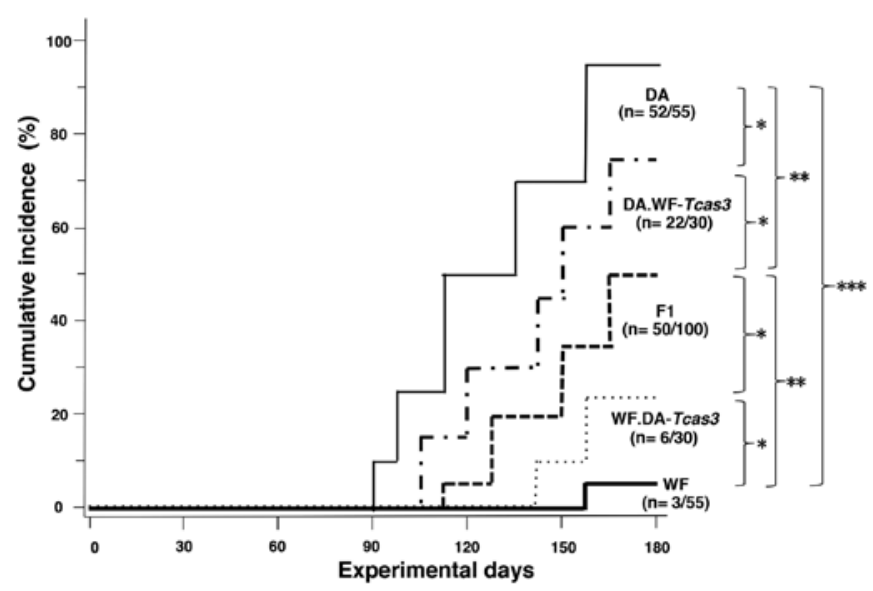

Figure 2. Cumulative incidence of 4NQO-induced tongue cancers (TC\#5, $>5 \mathrm{~mm}$ ). The solid line represents WF, the dotted line WF.DA-Tcas3, the dashed lines F1, the dotted/dashed lines DA.WF-Tcas3 and the thin solid line DA rats. ${ }^{*} \mathrm{P}<5 \times 10^{-2}$ significantly different from the corresponding values for WF and WF.DA-Tcas3, DA and DA.WF-Tcas $3 ;{ }^{* *} \mathrm{P}<5 \times 10^{-2}$ for DA and WF; F1 and WF; ${ }^{* * *} \mathrm{P}<1 \times 10^{-4}$ for WF and DA. 4NQO, 4-nitroquinoline 1-oxide; TC, tongue cancer; WF, Wistar-Furth rats; DA, Dark Agouti rats.

Effects of Tcas 3 on tongue carcinogenesis. We previously reported tumor incidence, number and size in descending order in DA, F1 and WF rats $(1,2,12)$. To evaluate the effect of introgressed Tcas 3 segments, rats of both congenic strains, parental DA and WF strains and F1, were administered 4NQO, and tongue and oral carcinogenesis was observed. As shown in Fig. 2, DA rats developed TCs with the shortest latency and highest incidence, whereas WF rats developed TCs with the longest latency and lowest incidence. In congenic strains, Tcas $3^{D A}$ showed modest but significant accelerating effects on TCs in WF rats, whereas Tcas $3^{W F}$ had TC-inhibitory effects on DA rats. These differences in strains were reflected by 
Table I. 4NQO-induced rat tongue and other cancers in the WF, F1, congenic and DA rats.

\begin{tabular}{|c|c|c|c|c|c|}
\hline & $\begin{array}{c}\text { WF } \\
(n=55)\end{array}$ & $\begin{array}{c}\text { Congenic } \\
\text { WF.DA-Tcas3 } \\
(\mathrm{n}=30)\end{array}$ & $\begin{array}{c}\mathrm{F} 1 \\
(\mathrm{n}=100)\end{array}$ & $\begin{array}{c}\text { Congenic } \\
\text { DA.WF-Tcas3 } \\
\quad(\mathrm{n}=30)\end{array}$ & $\begin{array}{c}\text { DA } \\
(n=55)\end{array}$ \\
\hline Rats with TC\#5 (incidence, \%) & $5.5(3 / 55)$ & $20(6 / 30)^{b}$ & $50(50 / 100)^{\mathrm{c}}$ & $73.3(22 / 30)^{\mathrm{c}}$ & $94.6(52 / 55)^{\mathrm{d}}$ \\
\hline No. of TC\#5 & $0.23 \pm 0.22$ & $0.33 \pm 0.12^{\mathrm{b}}$ & $0.61 \pm 0.71^{\mathrm{c}}$ & $0.68 \pm 1.20^{\mathrm{c}}$ & $1.28 \pm 1.56^{\mathrm{d}}$ \\
\hline Total no. of $\mathrm{TCs}^{\mathrm{a}}$ & $0.52 \pm 0.82$ & $0.75 \pm 0.58^{b}$ & $0.92 \pm 0.86^{c}$ & $1.01 \pm 0.76^{\mathrm{c}}$ & $1.59 \pm 0.66^{\mathrm{d}}$ \\
\hline DTCmax (mm) & $1.18 \pm 1.63$ & $2.12 \pm 3.54^{\mathrm{b}}$ & $5.15 \pm 1.85^{\mathrm{c}}$ & $5.89 \pm 1.17^{\mathrm{c}}$ & $12.2 \pm 4.49^{\mathrm{d}}$ \\
\hline Total no. of cancers ${ }^{a}$ & $1.26 \pm 1.45$ & $1.39 \pm 5.46^{\mathrm{b}}$ & $1.56 \pm 1.67^{\mathrm{c}}$ & $2.89 \pm 1.64^{\mathrm{c}}$ & $3.45 \pm 1.58^{\mathrm{d}}$ \\
\hline
\end{tabular}

4NQO, 4-nitroquinoline 1-oxide; TC, tongue cancer; WF, Wistar-Furth rats; DA, Dark Agouti rats. TC\#5, number of TCs $\geq 5$ mm in diameter. DTCmax, diameter of the largest TC. Total no. of cancers include TC and other cancers (the hard palate, pharynx, larynx, gingiva, trachea and

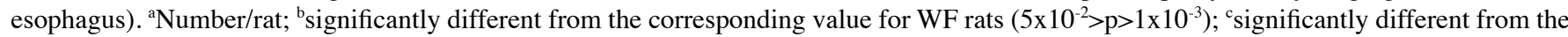
corresponding value for WF rats $\left(1 \times 10^{-3}>\mathrm{p}>1 \times 10^{-4}\right)$; ${ }^{\mathrm{d}}$ significantly different from the corresponding value for WF rats $\left(\mathrm{p}<1 \times 10^{-4}\right)$.
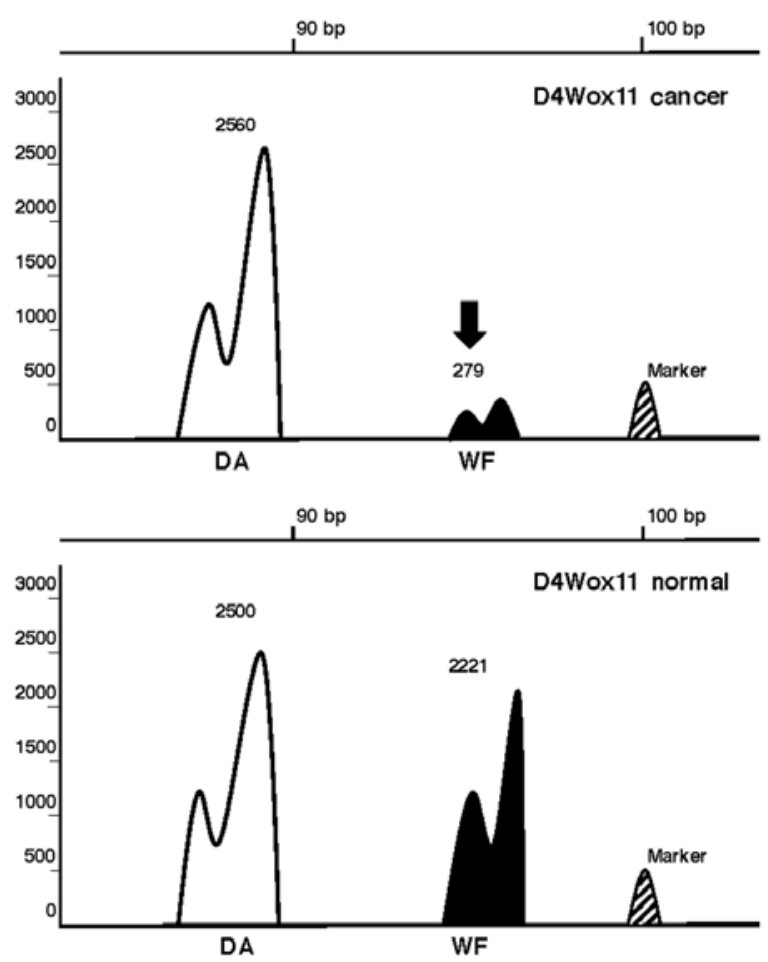
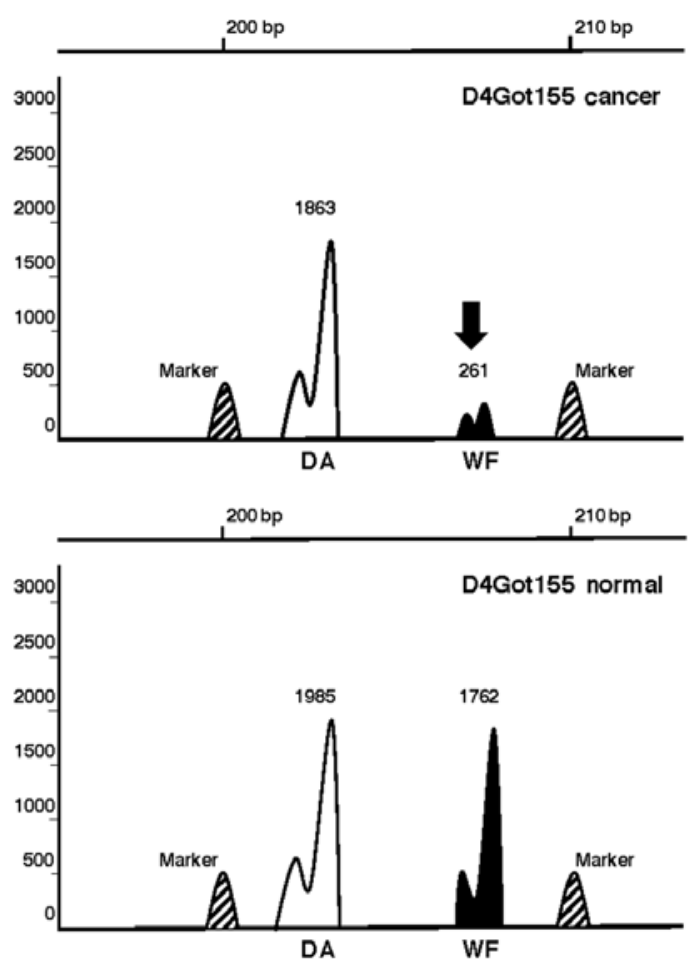

Figure 3. Representative LOH data analysis at microsatellite loci D4Wox11 (Pthlh) (left) and D4Got155 (right). The upper panels show the peak heights in arbitrary units of the WF (filled) and DA (unfilled) alleles from a TC in a (DA x WF) F1 rat, and the lower panels show those from the normal tongue tissue of the same rat. LOH, loss of heterozygosity; WF, Wistar-Furth rat; DA, Dark Agouti rat.

incidence, number and size of tumors (Table I). These observations clearly indicate that Tcas 3 alone exhibits significant phenotypic effects on the development and growth of TCs and oral cancers. That is $\operatorname{Tcas} 3^{D A}$ augmented grown whereas Tcas $3^{W F}$ suppressed it.

Selective LOH of Tcas $3^{W F}$ in larger TCs. To determine whether suppressive Tcas $3^{W F}$ was selectively deleted in TCs, genomic DNA of TCs induced in F1 rats was examined for LOH. Out of $100 \mathrm{~F} 1 \mathrm{rats}, 10 \mathrm{TCs}$ of $<5-\mathrm{mm}$ in diameter, $29 \mathrm{TCs}$ of $5-10 \mathrm{~mm}$ in diameter and 40 non-TC tissues did not show any LOH. However, in 21 TCs of $\geq 10-\mathrm{mm}$ diameter, $\mathrm{LOH}$ was observed between D4Mgh10 and D4Mgh13 on chromosome 4q44. In the majority of cases, the WF allele was selectively lost (Fig. 3). Among the major cancer-related genes on the Tcas3 segment, the incidence of LOH at Kras2 and Pthlh was 32.7 and $40.8 \%$, respectively in the TC\#5 (Table II). Loss of the Tcas $3^{W F}$ allele only in larger TCs indicates that it represents a late event in tumor progression.

Microarray analysis. To identify genes that are responsible for the effects of Tcas3, microarray analysis was performed in TCs and normal tongue tissues from F1 rats. According to the Rat Genome Database (6), 27 genes, 6 ESTs and 18 pseudogenes 
Table II. Immunostaining of the PTHrP protein in tongue cancer (TC) samples from F1 rats.

\begin{tabular}{|c|c|c|c|c|c|c|c|c|c|c|c|c|c|}
\hline \multirow{2}{*}{$\begin{array}{l}\text { Rat case } \\
\text { no. }\end{array}$} & \multirow{2}{*}{$\begin{array}{l}\text { DTCmax } \\
(\mathrm{mm})\end{array}$} & \multirow[b]{2}{*}{ TC\#5 } & \multicolumn{11}{|c|}{ Microsatellite markers ${ }^{\mathrm{a}}$ on $\mathrm{RNO} 4$} \\
\hline & & & Mgh30 & Mgh10 & ENO2 & Mit27 & Rat140 & Kras 2 & Got155 & Pthlh & Rat70 & Rat72 & Mghl3 \\
\hline 30 & 10 & 2 & - & - & - & - & - & - & - & WF & - & - & - \\
\hline 31 & 12 & 1 & - & - & - & - & - & - & - & WF & - & - & - \\
\hline 32 & 12 & 2 & - & - & - & - & - & - & WF & WF & - & - & - \\
\hline 33 & 13 & 1 & - & - & - & - & - & - & WF & WF & - & - & - \\
\hline 34 & 13 & 1 & - & - & - & - & - & WF & WF & WF & - & - & - \\
\hline 35 & 14 & 1 & - & - & - & - & - & WF & WF & WF & - & - & - \\
\hline 36 & 14 & 2 & - & - & - & - & - & WF & WF & WF & - & - & - \\
\hline 37 & 15 & 2 & - & - & - & - & - & WF & NI & WF & - & - & - \\
\hline 38 & 15 & 2 & - & - & - & - & - & WF & WF & WF & WF & - & - \\
\hline 39 & 15 & 2 & - & - & - & - & - & WF & WF & WF & WF & - & - \\
\hline 40 & 16 & 1 & - & - & - & - & - & NI & WF & WF & WF & - & - \\
\hline 41 & 17 & 2 & - & - & - & - & - & WF & WF & WF & WF & - & - \\
\hline 42 & 18 & 1 & - & - & - & - & - & WF & WF & WF & WF & - & - \\
\hline 43 & 19 & 2 & - & - & - & - & - & DA & DA & $\mathrm{DA}$ & WF & WF & - \\
\hline 44 & 20 & 3 & - & - & - & - & WF & WF & WF & WF & WF & WF & - \\
\hline 45 & 21 & 2 & - & - & - & - & WF & WF & WF & WF & WF & WF & - \\
\hline 46 & 22 & 2 & - & - & - & - & WF & WF & WF & $\mathrm{NI}$ & WF & WF & - \\
\hline 47 & 23 & 2 & - & - & - & - & WF & WF & WF & WF & WF & WF & - \\
\hline 48 & 24 & 2 & - & - & - & - & WF & WF & WF & WF & WF & WF & - \\
\hline 49 & 25 & 2 & - & - & - & WF & WF & WF & WF & WF & WF & WF & WF \\
\hline 50 & 25 & 3 & - & WF & WF & WF & WF & $\mathrm{WF}^{\mathrm{b}}$ & WF & WF & WF & WF & WF \\
\hline Incidence & & & $0 / 50$ & $1 / 50$ & $1 / 50$ & $2 / 50$ & $7 / 50$ & $16 / 49$ & $18 / 49$ & $20 / 49$ & $13 / 50$ & $8 / 50$ & $2 / 50$ \\
\hline Total $(\%)$ & & & 0 & 2.0 & 2.0 & 4.0 & 14.0 & 32.7 & 36.7 & 40.8 & 26.0 & 16.0 & 4.0 \\
\hline
\end{tabular}

RNO4, rat chromosome 4; 4NQO, 4-nitroquinoline 1-oxide; TC, tongue cancer. The lost alleles are denoted: WF, Wistar-Furth; DA, Dark Agouti; -, the presence of 2 alleles; NI, not informative. DTCmax, maximum diameter of the largest TC; TC\#5; number of TCs $\geq 5 \mathrm{~mm}$ in diameter.

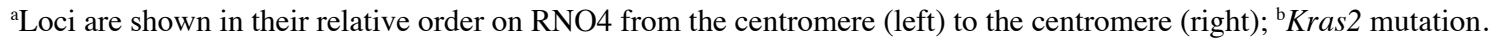

are present in the Tcas3 region. Table III shows the expression of the 27 genes as determined by microarray analysis. Significantly increased expression was observed for Pthlh (7.33-fold, $\mathrm{P}<0.001)$ and slightly less for Kras2 (5.21-fold, $\mathrm{P}<0.001)$.

The expression of genes in the Tcas 3 region was analyzed in 10 control and 60 TC samples using quantitative real-time RT-PCR. Pthlh mRNA expression in large TCs (>10 mm) was consistently $>30$-fold higher than that in normal tongue mucosa (Fig. 4), and expression levels were higher in larger tumors.

Sequencing of cancer-related genes in the Tcas3 segment. Pthlh and Kras 2 were further examined by sequencing germline and/or tumor DNA. Direct sequencing of the germline Pthlh gene was performed for 8 laboratory rat strains: DA, LE, SD, ACI, Fischer 344, Donryu, DRH and WF. The Pthlh gene in resistant WF rats was found to carry 3 SNPs at positions +2 bp $(\mathrm{T} \rightarrow \mathrm{G}),+17 \mathrm{bp}(\mathrm{T} \rightarrow \mathrm{C})$ and $+1485(\mathrm{~A} \rightarrow \mathrm{C})$ from the $5^{\prime}$ end of exon 1, but these were not observed in the other strains (Fig. 5). The base change at position 2 and 17 does not cause amino acid substitutions, whereas that at position +1485 [496 bp in the coding sequence (CDS)] bp would substitute the polar

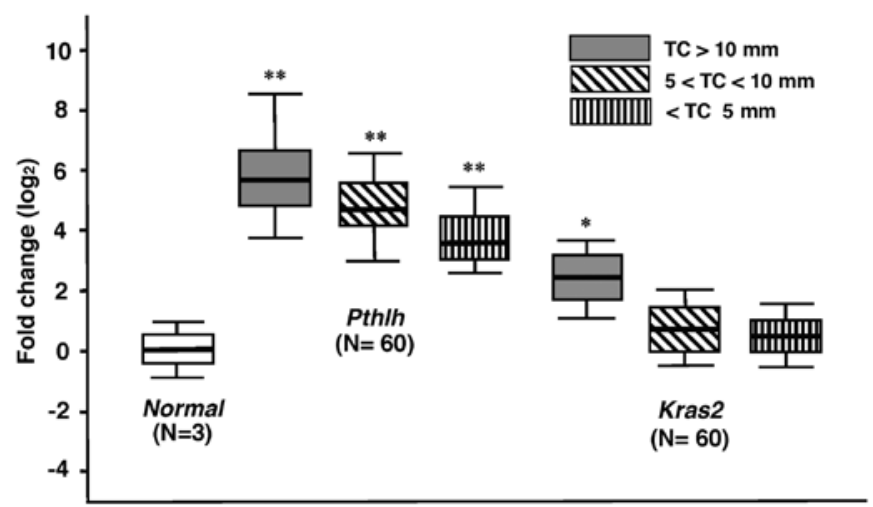

Figure 4. Quantitative analysis of Pthlh and Kras 2 mRNA levels in F1 tongue cancer (TC): TC $>10 \mathrm{~mm}$, TC 5-10 mm, TC $<5 \mathrm{~mm}$ and non-TC tissue F1 samples. The line within each box represents the median fold change. The upper and lower edges of each box represent the 75th and 25th percentiles, respectively. The upper and lower bars represent the highest and lowest value determined, respectively. ${ }^{* *} \mathrm{P}<0.001,{ }^{*} \mathrm{P}<0.01$.

residue threonine with the hydrophobic residue proline at the 166 th amino acid (ACC $\rightarrow \mathrm{CCC}$ ) of the precursor protein (posi- 
Table III. Microarray analysis of the Tcas3 region between tongue cancer and normal tissues in F1 rats.

\begin{tabular}{|c|c|c|c|c|}
\hline Symbol & Accession no. & Gene name & Fold change & P-value \\
\hline$A b c c 9$ & NM-013040.2 & ATP-binding cassette, subfamily $C(C F T R / M R P)$, member 9 & -1.58 & 0.413 \\
\hline Aebp2 & NM-001106626.1 & AE binding protein 2 & 0.23 & 0.625 \\
\hline Bcat1 & NM-017253.2 & Branched chain amino acid transaminase 1, cytosolic & -2.11 & 0.073 \\
\hline Bicdl & NM-001108653.1 & Bicaudal D homolog 1 & 2.31 & 0.071 \\
\hline Capza3 & NM-017164.1 & Capping protein (actin filament) muscle Z-line, $\alpha 3$ & -2.72 & 0.061 \\
\hline Cascl & BG377837.1 & Cancer susceptibility candidate 1 & 2.29 & 0.085 \\
\hline Cmas & NM-001009419.1 & Cytidine monophosphate N-acetylneuraminic acid synthetase & 1.71 & 0.561 \\
\hline Fgfrlop2 & NM-201421 & FGFR1 oncogene partner 2 & 2.89 & 0.051 \\
\hline Gys2 & NM-013089.1 & Glycogen synthase 2 & 1.12 & 0.761 \\
\hline Iapp & NM-012586 & Islet amyloid polypeptide & 0.56 & 0.982 \\
\hline Itpr 2 & NM-031046.3 & Inositol 1,4,5-trisphosphate receptor, type 2 & 1.26 & 0.781 \\
\hline Kcnj8 & NM-017099.4 & Potassium inwardly-rectifying channel, subfamily J, member 8 & 1.54 & 0.564 \\
\hline Kras2 & NM-031515.1 & Kirsten rat sarcoma viral oncogene homologue 2 & 5.21 & $<0.001$ \\
\hline$L d h b$ & NM-012595.1 & Lactate dehydrogenase $B$ & 1.89 & 0.647 \\
\hline Mgstl & NM-134349.2 & Microsomal glutathione S-transferase 1 & -2.54 & 0.062 \\
\hline$P d e 3 a$ & NM-017337.1 & Phosphodiesterase $3 A, c G M P$ inhibited & 1.98 & 0.653 \\
\hline$P i k 3 c 2 g$ & NM-053923.1 & Phosphatidylinositol-4-phosphate 3-kinase, catalytic subunit type $2 \gamma$ & 2.01 & 0.076 \\
\hline Plekha5 & AI009219.1 & Pleckstrin homology domain-containing protein family A, member 5 & 1.51 & 0.711 \\
\hline Pthlh & NM-012636.1 & Parathyroid hormone-like peptide & 7.33 & $<0.001$ \\
\hline Ptpro & NM-017336.1 & Protein tyrosine phosphatase, receptor type, $O$ & 1.09 & 0.881 \\
\hline Rassf8 & NM-001191753 & Ras association domain family ( $N$-terminal) member 8 & 2.97 & 0.051 \\
\hline Slc21a6 & NM-130736.1 & Solute carrier organic anion transporter family, member $1 a 6$ & 0.12 & 0.979 \\
\hline Slcola2 & NM-030838.1 & Solute carrier organic anion transporter family, member 1 A2 & 0.18 & 0.957 \\
\hline Slcolcl & NM-053441.1 & Solute carrier organic anion transporter family, member $1 c 1$ & 0.16 & 0.969 \\
\hline Sox5 & NM-001014060 & SRY (gender determining region Y)-box 5 & 1.11 & 0.652 \\
\hline St8sial & NM-012813.2 & ST8 $\alpha$-N-acetyl-neuraminide $\alpha$-2,8-sialyltransferase 1 & 1.87 & 0.489 \\
\hline$T m 7 s f 3$ & NM-001011970.1 & Transmembrane 7 superfamily member 3 & -2.32 & 0.065 \\
\hline
\end{tabular}

tion 130th of the mature protein) in which the CDS start is at 527 bp.

The potential impact of the 3 SNPs was determined using computational analyses. Since 2 SNPs at $+2 \mathrm{bp}(\mathrm{T} \rightarrow \mathrm{G})$ and $+17 \mathrm{bp}(\mathrm{T} \rightarrow \mathrm{C})$ were found in the untranslated region of the Pthlh gene, we searched for known transcription factor-binding sites within the -950 to $526 \mathrm{bp}$ region. TCF-4E (CTTTGCA) and ZFX (GAGGCCTGGTG) motifs were found at -1 to $+6 \mathrm{bp}$ and +11 to $+21 \mathrm{bp}$, respectively, in sequences from DA, LE, $\mathrm{SD}, \mathrm{ACI}, \mathrm{F} 344$, Donryu and DRH rats in which the +2 bp and 17 bp positions were occupied by $\mathrm{T}$. In the sequence from WF rats, PLU1-JARID1B.01 (TGGCTGTGC), SP1 (TGTGC) and ROAZ.01 (GTGCACCCAGAGGCCCG) motifs were found at -4 to +5 bp,+1 to +5 bp and +2 to +8 bp, respectively, with $\mathrm{G}$ and $\mathrm{C}$ residing at $+2 \mathrm{bp}$ and $+17 \mathrm{bp}$, respectively. The agreement rates in matrices of sequence motifs were 100, 98.8, 96.6, 100 and $73.1 \%$, respectively.

Using computational analyses, we evaluated the impact of the 166th amino acid substitution on the structure and function of the Pthlh protein. MedPred predicted a low impact, with a probability of deleterious mutation score of $0.134(>0.5$ indicates impact). Panther also predicted that the substitution was almost neutral, with a deleterious probability of $0.34315(>0.5$ indicates impact) and PhD-SNP predicted no impact. SNAP also predicted that the impact was neutral with $85 \%$ expected accuracy. Albeit with low confidence, SHIFT gave a score of 0 for affected protein function. Polyphen 2 did not yield a prediction. Thus, all bioinformatics tools revealed almost no impact of these SNPs on Pthlh protein structure and function.

Subsequently, Kras 2 point mutations were examined at codons 12, 13 and 61 in F1 TCs. One of the tumors had a heterozygous CAA $\rightarrow$ CAT mutation in codon 61 of Kras 2 , resulting in an amino acid substitution from glutamine to histidine. No other activating mutations were found in the Kras 2 gene. Therefore, any involvement of Kras 2 may not be predominant in rat TCs.

Plasma electrolyte and antibody levels in the WF, congenic and DA rats. Table IV shows the titer of plasma electrolytes and immunoreactive PTHrP in control and TC-bearing rats. Electrolyte levels in control rats did not vary according to genetic background and Tcas 3 genotype. However, plasma $\mathrm{Ca}^{2+}$ levels were consistently higher in TC-bearing rats than in control rats. We also evaluated levels of PTHrP peptides derived from post-translational cleavage of the whole protein. The PTHrP-C peptide levels were significantly elevated in 


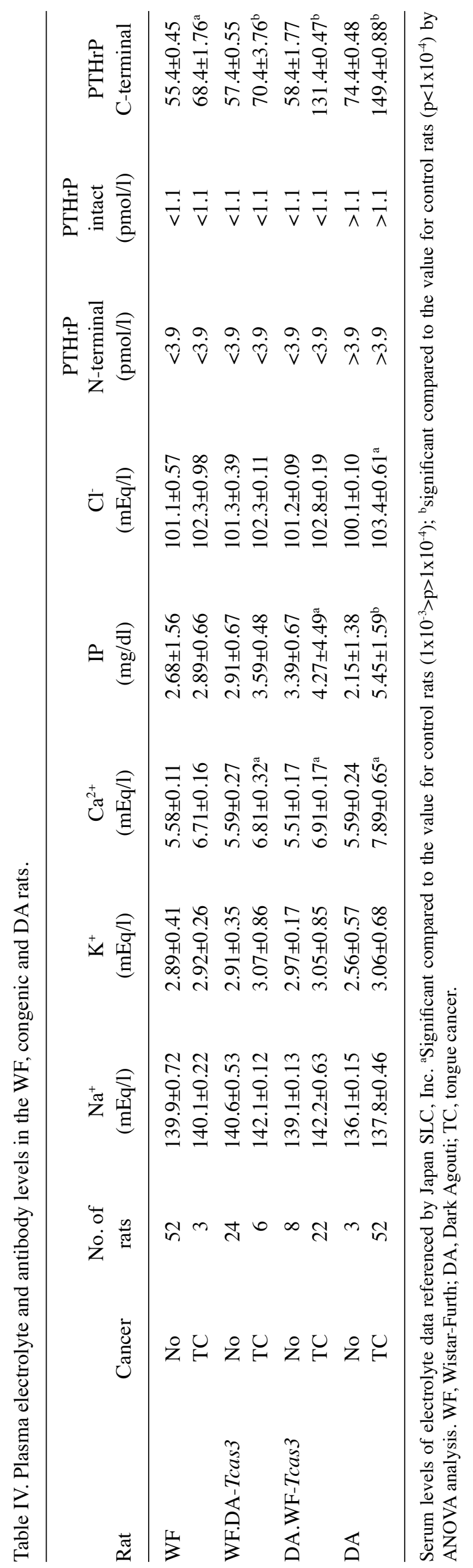

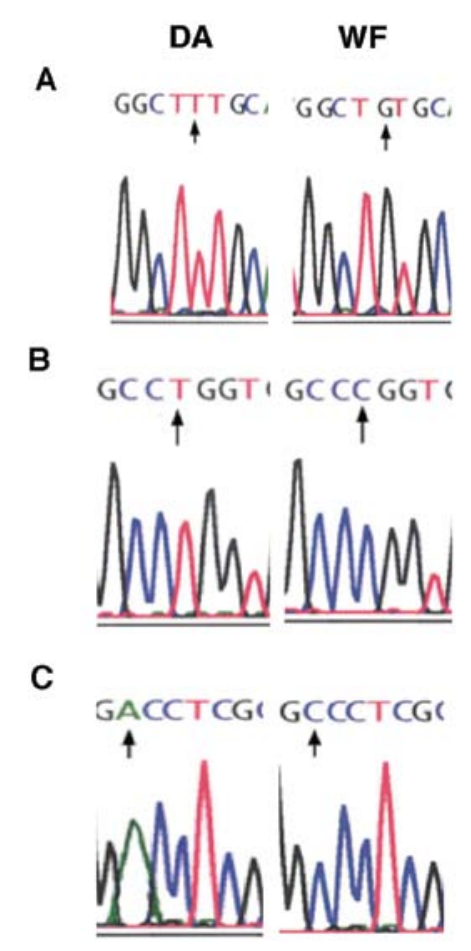

Figure 5. Detection of sequence variants in the Pthlh gene in the WF and DA strains. The WF strain contained 3 SNPs not noted in the DA and 6 other inbred strains of rat. The SNPs were located at positions (A) $+2 \mathrm{bp}$, (B) $+17 \mathrm{bp}$ and (C) $+513 \mathrm{bp}$. The sequences of the primers used for PCR amplification before sequencing were: Pthlh 1 F, 5'-GACTCGCTCACT TCTCAGCA-3' and R, 5'-GGCTCCCATAGCAATGTCTA-3'; Pthlh 2 F, 5'-GCGGTGTCTGAGCACCAGCTA-3' and R, 5'-GCACAGCGGACAGAC AATACC-3'. Primer pair 1 was used to amplify the SNPs at positions +2 and +17 bp; pair 2 amplified the SNP at position +1485 bp (B). WF, Wistar-Furth rats; DA, Dark Agouti rats.

TC-bearing rats, whereas levels of PTHrP-N and PTHrP-intact were unchanged.

TC immunohistochemistry. Among 50 TC\#5 and 10 rats with TCs of $<5 \mathrm{~mm}$ in F1 rats, positive signals for PTHrP were detected in the nucleus and cytoplasm of prickle-type cancer cells (Fig. 6). Staining intensity above or below the cut-off score (10\%) was classified as 'positive' or 'negative', respectively, at a magnification of x100 using computer-associated image analyzer software (WinROOF 7.1; Mitani Co., Japan) (26). As shown in Table V, the fraction of positively stained TCs increased with their size. There were significant differences in TCs between the TC\#5 and DTCmax groups $(\mathrm{P}<0.001)$, and among the TCs $<5 \mathrm{~mm}, 5-10 \mathrm{~mm}$ and TC $>10 \mathrm{~mm}\left(\mathrm{P}<1 \times 10^{-10}\right.$, $\left.\chi^{2}=47.63\right)$. The expression of Kras 2 was consistently weaker than that of PTHrP (data not shown).

\section{Discussion}

Out of 5 QTLs that affect susceptibility to TCs in 4NQO-treated rats, we focused on Tcas 3 in the present study. In genome-wide association studies in the F2 rat, the peak logarithm of the odds score 6.88 was observed for Tcas 3 at $4 \mathrm{q} 44$ (2). This chromosomal region is homologous to human 12p12.1-q11.2 and harbors the cancer-related genes Pthlh and Kras $2(7,12)$. To evaluate the effects of a single Tcas3, we generated speed congenic strains for Tcas 3 using marker-assisted backcrossing. 


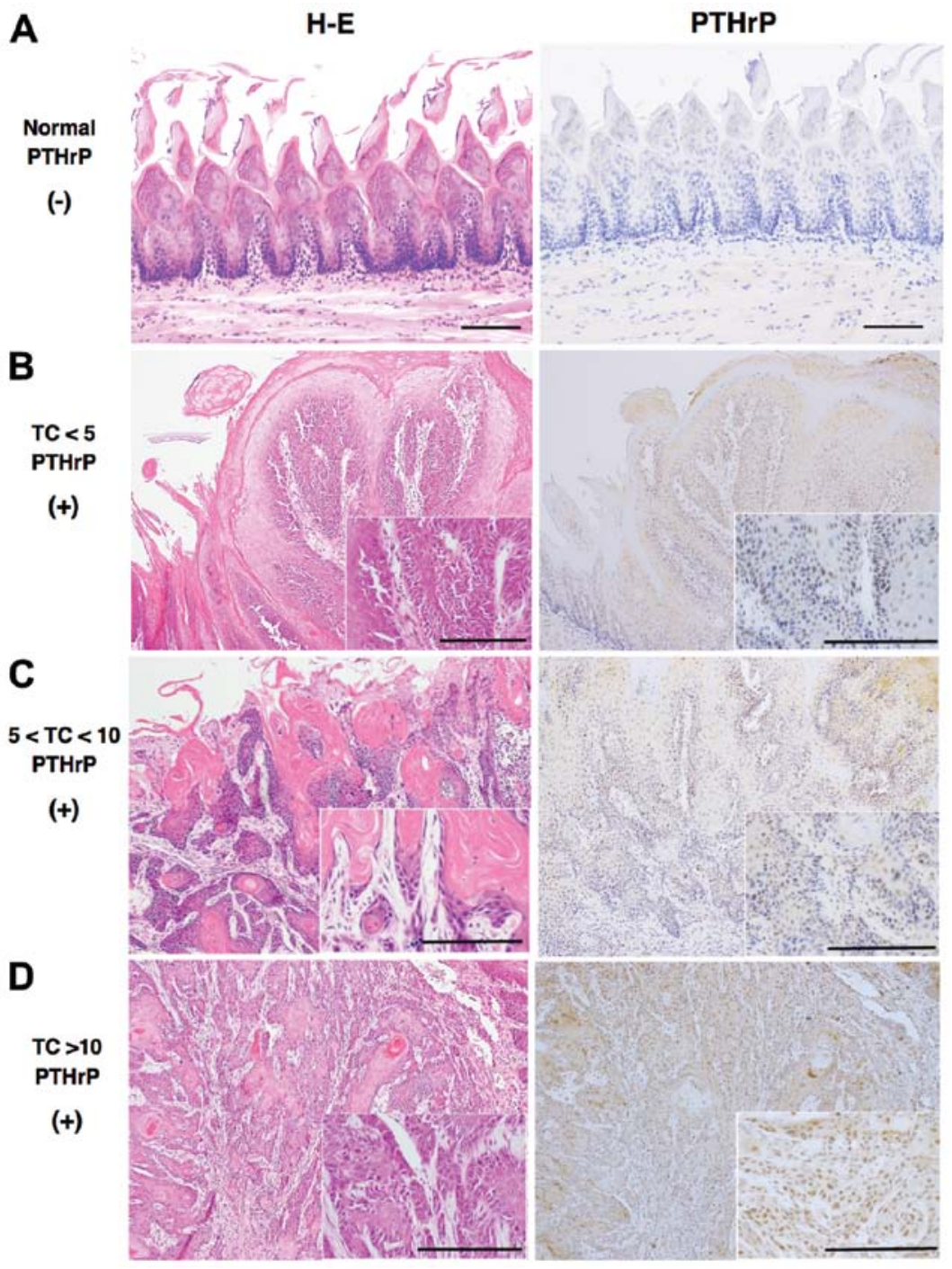

Figure 6. Hematoxylin and eosin (H\&E) staining (left) and anti-PTHrP immunohistochemistry (right) of F1 normal rat tongue (A) and tongue cancers (TC) $(<5 \mathrm{~mm}$ in diameter (TC <5) for H\&E and PTHrP-positive (+) (B); TC of 5-10 mm (TC 5-10) for H\&E and PTHrP (+) (C); TC $>10 \mathrm{~mm}(\mathrm{TC}>10)$ for H\&E and PTHrP (+) (D). The PTHrP-(+) tissue shows a positive signal in the cytoplasm and nucleus of many cancer cells. Scale bars, $200 \mu \mathrm{m}$.

Table V. Immunostaining of the PTHrP protein in tongue cancer (TC) samples from F1 rats.

\begin{tabular}{lcc}
\hline & Positive & Negative \\
Rat normal tissue $(\mathrm{n}=10)$ & 0 & 10 \\
TCs $<5 \mathrm{~mm}(\mathrm{n}=10)$ & 1 & 9 \\
TCs $5-10 \mathrm{~mm}(\mathrm{n}=29)$ & 24 & 5 \\
TCs $>10 \mathrm{~mm}(\mathrm{n}=21)$ & 21 & 0 \\
\hline
\end{tabular}

Staining intensity above or below the cut-off score (10\%) was classified as 'positive' or 'negative' at x100 magnification using a computer-associated image analyzer software (WinROOF 7.1). Significantly different from the corresponding values for F1 normal rats tissue $\left(\mathrm{P}<1 \times 10^{-10}, \chi^{2}=47.63\right)$.

As shown in Table I and Fig. 2, the quantitative parameters of carcinogenesis, namely tumor latency, incidence, tumor number and size, were moderately but significantly modified by Tcas3. Hence the Tcas $3^{D A}$ allele increased susceptibility to tongue carcinogenesis and progression, whereas the $T c a s 3^{W F}$ allele bestowed resistance. Selective loss of the resistance allele Tcas $3^{W F}$ was frequent in larger TCs induced in F1 rats, and the consensus stretch of LOH contained Pthlh and Kras2. Microarray comparisons of TCs and normal tongue mucosa also indicated that among genes in the Tcas 3 region these 2 genes were significantly highly expressed. We focused on Pthlh following the observation that resistant WF Pthlh rats carry 3 unique SNPs. The impact of these SNPs will be discussed in greater detail below. Other observations that suggest the relevance of Pthlh in tongue carcinogenesis include (i) a 30-fold higher expression of Pthlh in TCs than that in normal tongue epithelium, (ii) very strong PTHrP immonostaining in larger TCs from F1 rats and (iii) increased plasma levels of $\mathrm{Ca}^{2+}$ and PTHrP-C in TC-bearing DA rats when compared with the control rats.

The anabolic effects of Pthlh have been demonstrated in rodents and in humans. Pthlh is expressed in many cell types and usually acts as an autocrine, paracrine, and/or intracrine factor that plays numerous roles in embryonic development and physiology $(27,28)$. PTHrP may also have important 
functions in tumor development. PTHrP has been shown to stimulate proliferation and invasiveness of cancer cells as well as protection from apoptosis. $\mathrm{PTHrP}$ has the potential to cause malignant hypercalcemia and induce local osteolysis, which facilitates the growth of tumor cells that have metastasized to bone $(28,29)$. Partial homology of PTHrP and parathyroid hormone allows PTHrP to activate parathyroid hormone 1 receptor (PTH1R). PTH1R activation in bone and kidney leads to bone absorption and renal calcium retention, respectively, inducing a rise in blood calcium levels (30).

PTHrP consists of 3 molecular domains, the parathyroid hormone-like domain in the $\mathrm{N}$-terminal region, a mid-region and a C-terminal domain. Post-translational cleavage of the PTHrP protein allows these domains to function independently. The parathyroid hormone-like domain stimulates protein kinase $\mathrm{A}$, protein kinase $\mathrm{C}$, and/or the calcium-dependent pathways by activating PTH1R $(31,32)$. The mid-region domain can enter the nucleus since it carries a bipartite nuclear localization sequence at residues 88-91 and 102-106 and an importin $\beta$-binding site at residues 66-94 (33). After translocation into the nucleus, the PTHrP mid-region domain modulates gene expression by an as yet undefined mechanism. Nuclear transport of the mid-region domain is regulated by CDK1(CDC2)/CDK2-dependent phosphorylation (34). The C-terminal domain of PTHrP (osteostatin) physically interacts with $\beta$-arrestin (35), which regulates internalization and desensitization of ligand-stimulated G-protein-coupled receptors (36). Osteostatin bears a number of phosphorylation sites that are important for the mitogenic activity of PTHrP in vascular smooth muscle cells (37).

To understand the potential impact of SNPs in untranslated regions of PTHrP genes, we performed in silico analyses using computational tools with various prediction algorithms and found several known regulatory motifs on the 5'-UTR of the Pthlh sequence. These analyses identified only 2 transcription factors, TCF-4E and ZFX, in all strains apart from WF, indicating that the SNPs at +2 bp $(\mathrm{T} \rightarrow \mathrm{G})$ and +17 bp $(\mathrm{T} \rightarrow \mathrm{C})$ eliminate the transcriptional factor binding site. In contrast, PLU1-JARID1B.01, SP1 and ROAZ.01 were found only in WF rats, indicating that these 2 SNPs affect transcription factor binding to these motifs. ZFX and ROAZ.01 are zinc finger proteins with a DNA-binding motif, and SP1 is common to many eukaryotic transcriptional regulatory pathways. The transcription factor TCF-4E regulates expression of members of the Wnt pathway and its splicing isoforms are related to the progression of renal cell carcinoma (38). The motif PLU1-JARID1B.01 was previously identified in the development and progression of breast cancer (39). However, no clear relationship between these transcription factors and oral cancer development has been reported to date. Hence, further research is necessary to understand the effect of these motifs and SNPs at +2 and +17 bp. Using several bioinformatics tools with varying predictive algorithms, we also analyzed structural and functional effects of the SNP at $+1485 \mathrm{bp}$, which leads to an amino acid substitution at the 166th amino acid on the Pthlh protein. Notably, none of these analyses suggested that this SNP would have any effect. Potentially, these computational tools, which are generated using characterized protein, may not predict novel functional motifs, even though they yielded consistent predictions. Benelli et al (15) reported an amino acid polymorphism in Pthlh that elicited cancer-modifying effects in a mouse squamous cell carcinoma cell line (15). Importantly, the SNP at position +1485 [+496 bp in the coding sequence (CDS)] bp was common to both mouse and rat models. This SNP can be expected to substitute the polar residue threonine with the hydrophobic residue proline at the 166th amino acid (ACC $\rightarrow \mathrm{CCC}$ ) of the precursor protein (position 130th of the mature protein), in which the CDS start is at $527 \mathrm{bp}$.

The present study revealed that Tcas 3 alone contributes moderate but significant phenotypic effects to 4NQO-induced rat tongue carcinogenesis. Several observations suggest that Pthlh is a promising candidate gene in Tcas3, although further studies using appropriate reporter assays and in vivo transgenic analyses are required to provide direct supporting evidence of this. In subsequent studies, we will focus on structural and functional analyses of Pthlh and its products.

\section{Acknowledgements}

The present study was supported by a grants-in-aid for Scientific Research (C) from the Japan Society for Promotion of Science (no. 24592850).

\section{References}

1. Tanuma J, Shisa H, Hiai H, et al: Quantitative trait loci affecting 4-nitroquinoline 1-oxide-induced tongue carcinogenesis in the rat. Cancer Res 58: 1660-1664, 1998.

2. Tanuma J, Fujii K, Hirano M, et al: Five quantitative trait loci affecting 4-nitroquinoline 1-oxide-induced tongue cancer in the rat. Jpn J Cancer Res 92: 610-616, 2001.

3. Kitano M, Hatano H and Shisa H: Strain difference of susceptibility to 4-nitroquinoline 1-oxide-induced tongue carcinoma in rats. Jpn J Cancer Res 83: 843-850, 1992.

4. Kitano M, Hirayama Y, Tanuma J, et al: Genetic controls of susceptibility and resistance to 4-nitroquinoline 1-oxide-induced tongue carcinomas in rats. Jpn J Cancer Res 87: 1097-1101, 1996.

5. Kwitek AE, Gullings-Handley J, Yu J, et al: High-density rat radiation hybrid maps containing over 24,000 SSLPs, genes, and ESTs provide a direct link to the rat genome sequence. Genome Res 14: 750-757, 2004.

6. RGD-Rat Genome Database. http://rgd.mcw.edu/ (May 1, 2011).

7. Tanuma J, Hirano M, Hirayama Y, et al: Genetic predisposition to $4 \mathrm{NQO}$-induced tongue carcinogenesis in the rat. Med Princ Pract 14: 297-305, 2005.

8. Hirano M, Tanuma J, Hirayama Y, et al: A speed congenic rat strain bearing the tongue cancer susceptibility locus $T s c c 1$ from Dark-Agouti rats. Cancer Lett 231: 185-191, 2006.

9. NBRP: The National BioResource Project for the Rat in Japan: NBRP Rat No. 0268. http://www.nbrp.jp/ (Aug 10, 2010).

10. Liu H, Higashi K and Hiai H: Role of resistant Drh1 locus in chemical carcinogen-induced hepatocarcinogenesis in rats: analysis with a speed congenic strain. Cancer Sci 96: 164-169, 2005.

11. Walentinsson A and Levan G: Ras gene mutations in 7,12-dimethylbenz[a]anthracene (DMBA)-induced rat sarcomas. Cancer Lett 166: 47-53, 2001.

12. Tanuma J, Hiai $\mathrm{H}$, Shisa $\mathrm{H}$, et al: Carcinogenesis modifier loci in rat tongue are subject to frequent loss of heterozygosity. Int $\mathbf{J}$ Cancer 102: 638-642, 2002.

13. Ogawa K, Tanuma J, Hirano M, et al: Selective loss of resistant alleles at $p 15^{I N K 4 B}$ and $p 16^{I N K 4 A}$ genes in chemically-induced rat tongue cancers. Oral Oncol 42: 710-717, 2006.

14. Manenti G, Peissel B, Gariboldi M, et al: A cancer modifier role for parathyroid hormone-related protein. Oncogene 19: 5324-5328, 2000.

15. Benelli R, Peissel B, Manenti G, et al: Allele-specific patterns of the mouse parathyroid hormone-related protein: influences on cell adhesion and migration. Oncogene 22: 7711-7715, 2003.

16. Li B, Krishnan VG, Mort ME, et al: Automated inference of molecular mechanisms of disease from amino acid substitutions. Bioinformatics 25: 2744-2750, 2009. 
17. Thomas PD, Campbell MJ, Kejariwal A, et al: PANTHER: a library of protein families and subfamilies indexed by function. Genome Res 213: 2129-2141, 2003.

18. Capriotti E, Calabrese R and Casadio R: Predicting the insurgence of human genetic diseases associated to single point protein mutations with support vector machines and evolutionary information. Bioinformatics 22: 2729-2734, 2006.

19. Bromberg Y and Rost B: SNAP: predict effect of non-synonymous polymorphisms on function. Nucleic Acids Res 35: 3823-3835, 2007.

20. Adzhubei IA, Schmidt S, Peshkin L, et al: A method and server for predicting damaging missense mutations. Nat Methods 7: 248-249, 2010

21. Ng PC and Henikoff S: Predicting deleterious amino acid substitutions. Genome Res 11: 863-874, 2001

22. Cartharius K, Frech K, Grote K, et al: Matlnspector and beyond: promoter analysis based on transcription factor binding sites. Bioinformatics 21: 2933-2942, 2005.

23. Schug J: Using TESS to transcription factor binding sites in DNA sequence. Curr Protec Bioinfomatics: 21: 2.6.1-2.6.15, 2008.

24. Laboratory animal data. Japan SLC Inc., 2007. http:// jslc.co.jp (Feb 2, 2011).

25. Fukunaga M, Eto S and Saito S: Multicentric clinical studies on measurement of carboxyl-terminal parathyroid hormone-related protein in normal subjects (the first report). Clin Endocrinol 40: 977-986, 1992

26. Takeda T, Sugihara K, Hirayama Y, et al: Immunohistological evaluation of Ki-67, p63, CK19 and p53 expression in oral epithelial dysplasias. J Oral Pathol Med 35: 369-375, 2006.

27. Dittmer J: The importance of PTHrP for cancer development. Gene Ther Mol Biol 8: 451-464, 2004.

28. Goltzman D, Karaplis AC, Kremer R and Rabbani SA: Molecular basis of the spectrum of skeletal complications of neoplasia. Cancer 88 (Suppl 12): 2903-2908, 2000.

29. Käkönen SM and Mundy GR: Mechanisms of osteolytic bone metastases in breast carcinoma. Cancer 97 (Suppl 3): 834-839, 2003.
30. Mannstadt M, Jüppner H and Gardella TJ: Receptors for PTH and PTHrP: their biological importance and functional properties. Am J Physiol 277: F665-F675, 1999.

31. Maioli E and Fortino V: The complexity of parathyroid hormone-related protein signaling. Cell Mol Life Sci 61: 257-262, 2004.

32. Cataisson C, Lieberherr M, Cros M, et al: Parathyroid hormonerelated peptide stimulates proliferation of highly tumorigenic human SV40-immortalized breast epithelial cells. J Bone Miner Res 15: 2129-2139, 2000.

33. Henderson JE, Amizuka N, Warshawsky H, et al: Nucleolar localization of parathyroid hormone-related peptide enhances survival of chondrocytes under conditions that promote apoptotic cell death. Mol Cell Biol 15: 4064-4075, 1995.

34. Lam MH, House CM, Tiganis T, et al: Phosphorylation at the cyclin-dependent kinases site $\left(\mathrm{Thr}^{85}\right)$ of parathyroid hormonerelated protein negatively regulates its nuclear localization. J Biol Chem 274: 18559-18566, 1999.

35. Conlan LA, Martin TJ and Gillespie MT: The $\mathrm{COOH}$-terminus of parathyroid hormone-related protein (PTHrP) interacts with $\beta$-arrestin 1B. FEBS Lett 527: 71-75, 2002.

36. Ferrari SL, Behar V, Chorev M, et al: Endocytosis of ligandhuman parathyroid hormone receptor 1 complexes is protein kinase C-dependent and involves $\beta$-arrestin2. Real-time monitoring by fluorescence microscopy. J Biol Chem 274: 29968-29975, 1999.

37. Fiaschi-Taesch N, Takane KK, Masters S, et al: Parathyroidhormone-related protein as a regulator of $\mathrm{pRb}$ and the cell cycle in arterial smooth muscle. Circulation 110: 177-185, 2004.

38. Shiina H, Igawa M, Breault J, et al: The human T-cell factor-4 gene splicing isoforms, Wnt signal pathway, and apoptosis in renal cell carcinoma. Clin Cancer Res 9: 2121-2132, 2003.

39. Scibetta AG, Santangelo S, Coleman J, et al: Functional analysis of the transcription repressor PLU-1/JARID1B. Mol Cell Biol 27: 7220-7235, 2007. 